\title{
Matrix Arrangement of Three-Dimensional Sheath Flow for Multiple Component Nanofibers
}

\author{
Dong Hyun Yoon, ${ }^{1}$ Asahi Nakahara, ${ }^{1}$ Afshan Jamshaid, ${ }^{1}$ Hironobu Sato, ${ }^{1}$ \\ Tetsushi Sekiguchi, ${ }^{2}$ and Shuichi Shoji ${ }^{1}$ \\ ${ }^{1}$ Faculty of Science and Engineering, Waseda University, 3-4-1 Okubo, Shinjuku-ku, Tokyo 169-8555, Japan \\ ${ }^{2}$ Institute for Nanoscience and Nanotechnology, Waseda University, 513 Tsurumaki-cho, Waseda, Shinjuku-ku, Tokyo 162-0041, Japan \\ Correspondence should be addressed to \\ Tetsushi Sekiguchi; t-sekiguchi@waseda.jp
}

Received 26 September 2012; Accepted 1 February 2013

Academic Editor: Takahiro Arakawa

Copyright (C) 2013 Dong Hyun Yoon et al. This is an open access article distributed under the Creative Commons Attribution License, which permits unrestricted use, distribution, and reproduction in any medium, provided the original work is properly cited.

Multiple core-sheath flow was realized using matrix arrangement of 3D sheath flows. The sheath flow was hydrodynamically formed in a flow shift area which has symmetrical microgrooves on channel walls. Vertical and horizontal alignments of the sample streams are a key element of matrix configuration. The flow shift areas were connected in parallel to achieve horizontal alignment of the sheath flow. The cascade connection of the flow shift areas is used for vertical alignment of the sheath flow. In order to achieve matrix arrangement of core-sheath flow, combination of the parallel and cascade connections was utilized. In this work, the horizontal and vertical configurations of the 2-sample sheath flow were demonstrated. Two streams of the vertically aligned 2-sample sheath flow were joined horizontally, and, as a result, 4-sample core-sheath flow of matrix configuration was obtained successfully.

\section{Introduction}

Nanofiber is a potentially useful material in various fields [1], and electrospinning is a simple way to fabricate nanofiber. In this method, syringe and needle are commonly used to feed materials in a high electric field. Control of properties of nanofiber, such as physical, chemical, and mechanical properties, is one of the key issues for practical application. Multiple component nanofiber which composed of various kinds of materials with different properties is effective in achieving control of properties. In order to obtain multicomponent nanofibers by electrospinning, several kinds of materials should be transferred at the same time to the electric field without mixing. However, it is difficult for a syringe to transfer all the samples separate from each other. Emulsion has been utilized to fabricate bicomponent fibers [2]. However, because of discontinuity of the droplet, the formed bicomponent region is intermittent and, considering multicomponent, it is very difficult to inject multiple kinds of droplets simultaneously to the high electric field. To realize multiple component fibers by using emulsion, the size, position, and timing of each emulsion droplets should be precisely controlled.

Microfluidic devices have been used to fabricate nano/microscale fibers [3-6]. Laminar flow condition in microchannel is effective in transferring several samples at the same time without mixing. Microfluidic chips have been applied to electrospinning, and continuous bicomponent nanofibers are successfully fabricated $[5,6]$. For considering biomedical applications, multicomponent nanofibers with a multiple core-shell configuration are preferable. For example, biocompatible materials of shell region can prevent poor biocompatible materials of core region from being exposed. 3D sheath flow is a suitable way to form core-shell configuration of fiber. Several 3D sheath flow devices have been reported [7-10]. Multilayer structure and multiple sheath inlets were popular methods. In order to achieve multiple sheath flow device, the part of the microstructure that enables coaxial sheathing of sample stream should be integrated into a chip. However, due to the complexity of those methods' structure 


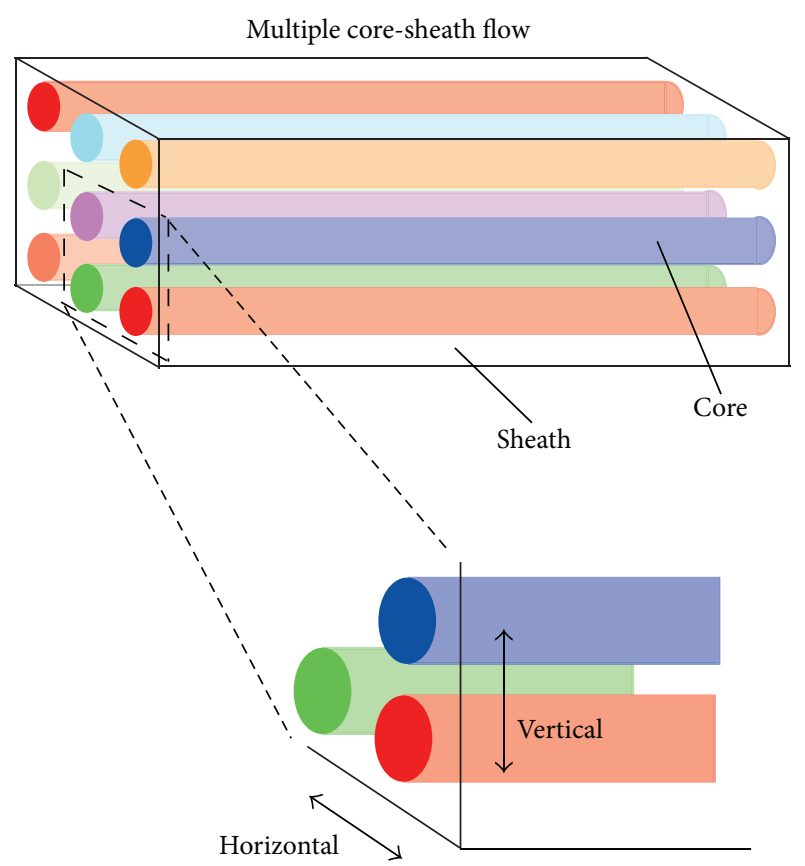

FIgURE 1: The concept of multiple core-sheath flow.

and increment of number of inlets, thes are not practical to realize multiple core-sheath flow devices. Recently, 3D sheath flow was hydrodynamically formed by using a 3D-grooved microchannel [11, 12]. Since these sheath flow devices have a very simple structure and a few sheath inlets, they are easy to integrate. So this method is preferable for developing multisample sheath flow devices.

\section{Principle}

2.1. Concept of Multiple Core-Sheath Flow. Figure 1 shows concept of multiple core-sheath flow. Several sample (core) flows are embedded in a carrier (sheath) flow. In order to achieve symmetry of fiber structure and core arrangement, sample streams are aligned in matrix. The matrix arrangement of sample stream is composed of horizontal and vertical alignments of sheath flows.

In this work, firstly, we developed horizontal and vertical configurations of sheath flows. Then the vertically aligned sheath flows are aligned horizontally to achieve matrix configuration of core-sheath flow. As demonstrations, the horizontal and vertical alignments of 2-sample sheath flow were developed. In horizontal sheath flow, control of sample flow rates was performed for potential application of control of the composition ratio of nanofiber. And 4-sample coresheath flow was realized by horizontal alignment of the two streams of the vertically aligned 2-sample sheath flows.

2.2. Three-Dimensional Sheath Flow. A 3D sheath flow is achieved by hydrodynamic control of the sample flow shift. $3 \mathrm{D}$ symmetrical microgrooves are formed on walls of the microchannel of the flow shift area. The slanted microgrooves are on the side walls, and V-shaped microgrooves are on the

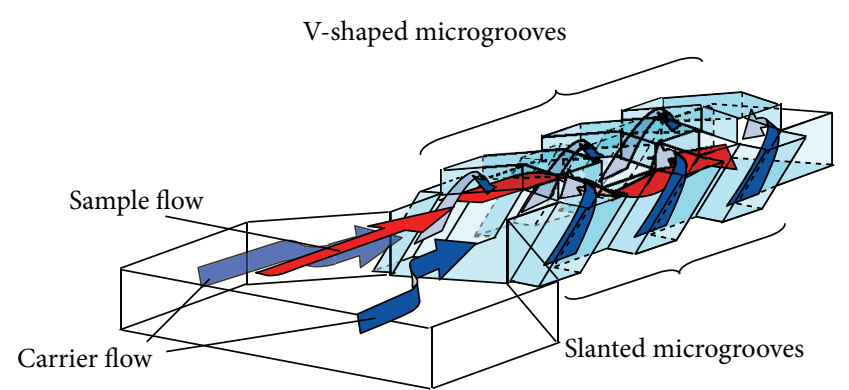

(a)

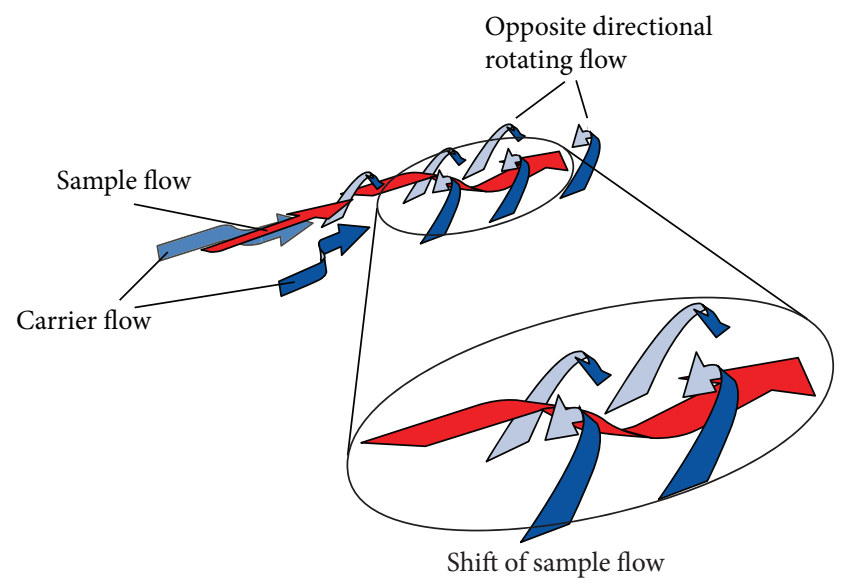

(b)

FIgURE 2: The schematic drawing of the flow shift behaviour.

top walls of the microchannel. Figure 2 shows the schematic drawing of the flow shift behavior. Since liquid flows along the symmetric microgrooves, two opposite directional rotating flows are generated, and at the center region of the microchannel cross-section downward stream is generated. The position of the sample flow is shifted by exploiting the downward stream to the center of the microchannel. As a result, the sample stream is completely surrounded by carrier stream, and a 3D sheath flow is realized. The grooved microchannel was formed in PDMS single layer, and only two inlets, one sample inlet and one sheath inlet, were needed. The flow shift areas could be easily integrated because of simple structure. The horizontal and vertical configurations of sheath flows were achieved by connecting the flow shift areas in parallel and cascade, respectively.

\section{Methods and Materials}

3.1. Device Fabrication Method. The sheath flow device was fabricated by soft lithography of PDMS using SU-8 structure as a mold. Figure 3 shows fabrication process of the SU8 mold. Firstly, SU-8 was spin coated on a Cr-patterned glass substrate, and UV was exposed to form microchannel (Figure 3(a)). In order to fabricate symmetric-slanted microgrooves on side walls, UV was irradiated from backside of the inclined substrate (Figure 3(b)). After spin coating of SU-8 second layer, UV was exposed to form V-shaped 
microgrooves on top of the mold (Figure 3(c)). Finally, SU-8 was developed and 3D mold structure was obtained (Figure 3(d)). The PDMS-molded structure was sealed by a glass substrate using oxygen plasma treatment.

3.2. Experimental Setup. 3\% polyvinylalcohol- (PVA-) water solution dyed with Rhodamine B and FITC (fluorescein isothiocyanate) were used as sample liquids. PVA-water solution without fluorescent dye was used as a carrier liquid. The sample and carrier liquid were introduced into a microchannel using syringe pumps (KDS200: Kd Scientific). 3D sheath flow was observed by cross-sectional fluorescent images acquired by a confocal fluorescent microscope (TCS SL: Leica Microsystems).

\section{Results and Discussions}

4.1. Flow Shift by 3D Structure. Figure 4 shows sample flow behavior in the flow shift area. Sample and carrier were introduced at the volume flow rate of $1 \mu \mathrm{L} / \mathrm{min}$ and $20 \mu \mathrm{L} / \mathrm{min}$, respectively. As mentioned above, it was observed that the sample stream gradually shifted toward the center of the microchannel, and at the end of the flow shift area the 3D sheath flow developed.

4.2. Horizontally Arrayed Sheath Flow. To achieve horizontally arrayed sheath flow configuration, the flow shift areas are simply connected in parallel. In this paper, two flow shift areas are connected to demonstrate a 2-sample sheath flow. The two streams of 3D sheath flow were joined, and a laterally arrayed 2 -sample sheath flow was achieved as shown in Figure 5. The ratio between the two samples of Rhodamine B and FITC can be controlled by changing the flow rate. Each flow rate was controlled from $1 \mu \mathrm{L} / \mathrm{min}$ to $5 \mu \mathrm{L} / \mathrm{min}$; meanwhile, the total flow rate was constant at $42 \mu \mathrm{L} / \mathrm{min}$. It was observed that the diameter of each samples changed along with its flow rate. The control of the sample ratio is potentially applicable to realize changing of the composition ratio of nanofiber.

4.3. Vertically Arrayed Sheath Flow. To achieve vertically arrayed sheath flow configuration, the flow shift area was connected in cascade. As a demonstration, two flow shift areas were connected, and a vertically aligned two-sample sheath flow was obtained, as shown in Figure 6. The flow rate of Rhodamine B and FITC was $1 \mu \mathrm{L} / \mathrm{min}$ each, and the total flow rate was $22 \mu \mathrm{L} / \mathrm{min}$. In cascade connection, one carrier inlet is needed and total flow rate is lower than that of parallel connection. Though total flow rate is different compared to that of the parallel connection, the flow shift behavior is the same because of laminar flow regime. It is also observed that the diameter of the sample in the channel cross section changed along with the flow rate. Since the degree of the sample flow shift depends on the number of the microgrooves formed on channel walls, the distance between the two samples can be controlled through the combination of the number of the microgrooves between the former and the latter flow shift areas.
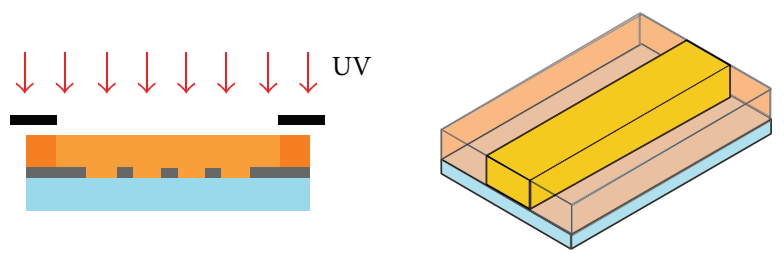

UV exposure to form microchannel

(a)
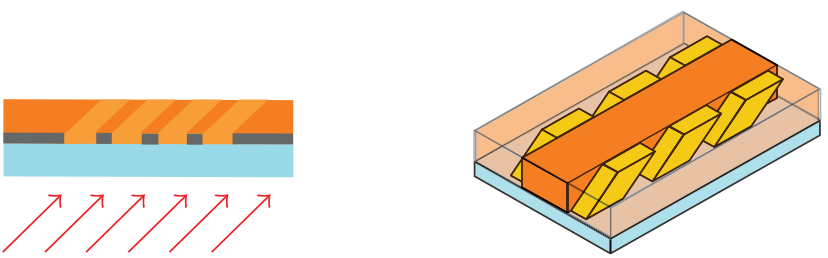

Inclined backside UV exposure to form slanted microgrooves on side walls of the microchannel

(b)

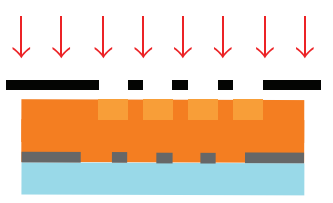

UV exposure to form V-shaped microgrooves on top walls of the microchannel

(c)

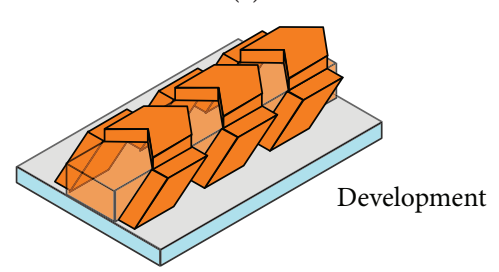

(d)

FIGURE 3: The fabrication process of the SU-8 mold.

4.4. Multiple Core-Sheath Flow. Multiple core-sheath flow can be realized by combining the parallel and cascade connections of the flow shift areas. In this work, two vertically aligned 2-sample sheath flows were laterally arrayed side by side to realize a 4 -sample core-sheath flow. Two cascade connections of the flow shift area were connected in parallel. The flow rate of the samples was $1 \mu \mathrm{L} / \mathrm{min}$ and that of a carrier flow was $20 \mu \mathrm{L} / \mathrm{min}$; as a result, total flow rate was $44 \mu \mathrm{L} / \mathrm{min}$. Figure 7 shows 4 -sample core-sheath flow. It was observed that each sample flow was vertically elongated, and the separation between Rhodamine B and FITC became unclear. In this device, two branches of the 2-sample sheath flow microchannel were joined into a main microchannel. Since the dimension of these microchannels was the same (width: $100 \mu \mathrm{m}$, height: $100 \mu \mathrm{m}$ ), each sheath flow should be compressed in lateral; as a result, the sample flows of Rhodamine B and FITC were elongated in vertical direction and their boundary became unclear. To realize a more clearly separated core-sheath flow, the width of the main channel 


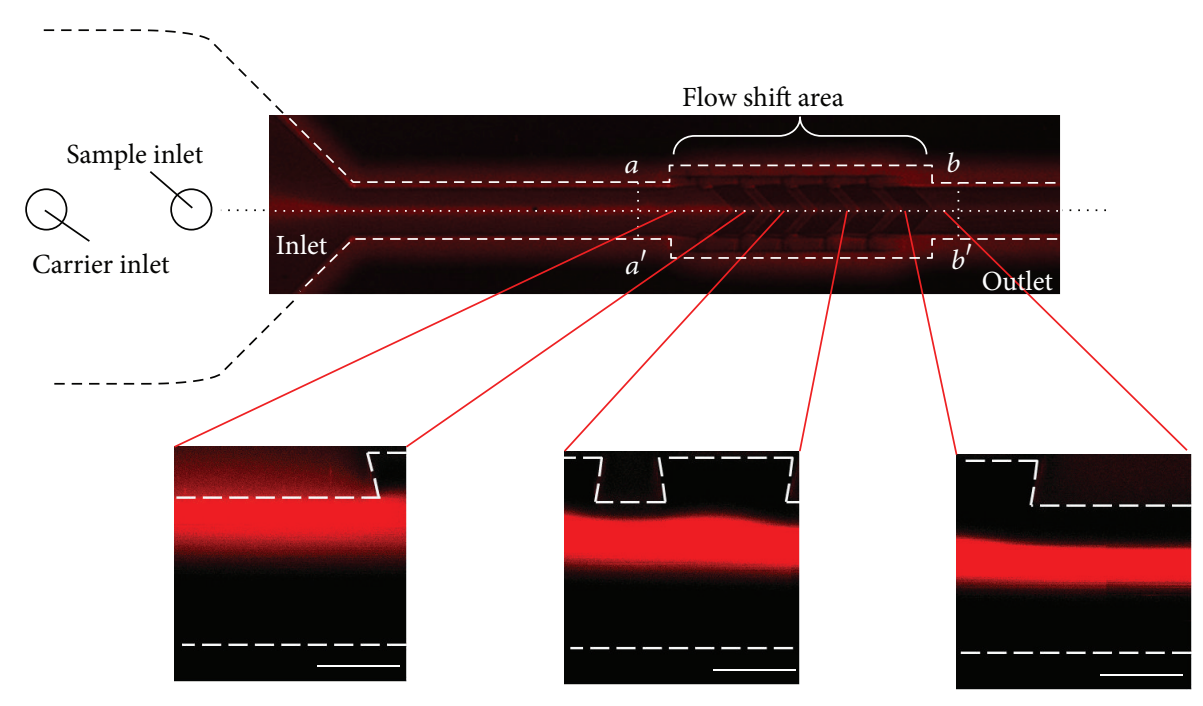

Lengthwise cross-sectional images

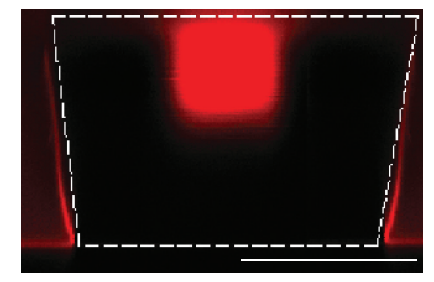

$a-a^{\prime}$ cross section

(entrance of the flow shift area)

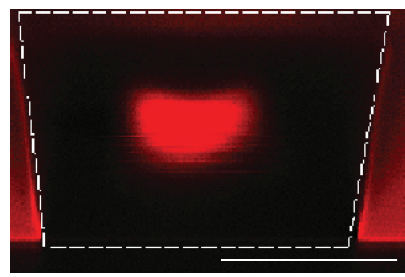

$b-b^{\prime}$ cross section (exit of the flow shift area)

FIgURE 4: The sample flow behavior in a flow shift area. Scale bar is $50 \mu \mathrm{m}$.

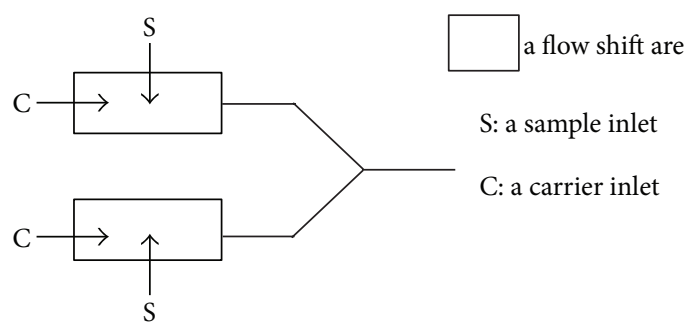

(a)
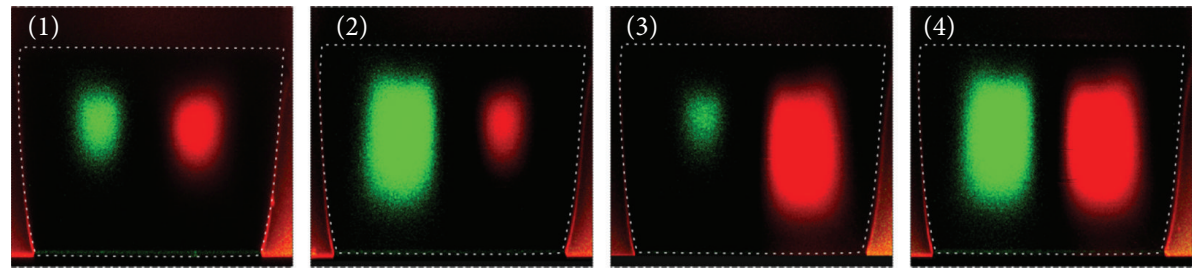

(b)

Figure 5: The horizontally arrayed sheath flow. (a) Parallel connection of the flow shift areas. (b) Horizontally arrayed 2-sample sheath flow. Scale bar is $50 \mu \mathrm{m}$. (1) FITC is $1 \mu \mathrm{L} / \mathrm{min}$. Rhodamine B is $1 \mu \mathrm{L} / \mathrm{min}$. (2) FITC is $5 \mu \mathrm{L} / \mathrm{min}$. Rhodamine B is $1 \mu \mathrm{L} / \mathrm{min}$. (3) FITC is $1 \mu \mathrm{L} / \mathrm{min}$. Rhodamine B is $5 \mu \mathrm{L} / \mathrm{min}$. (4) FITC is $5 \mu \mathrm{L} / \mathrm{min}$. Rhodamine B is $5 \mu \mathrm{L} / \mathrm{min}$. 


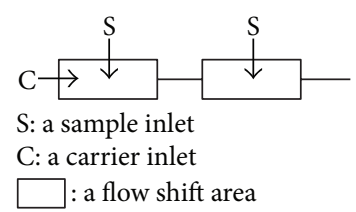

(a)

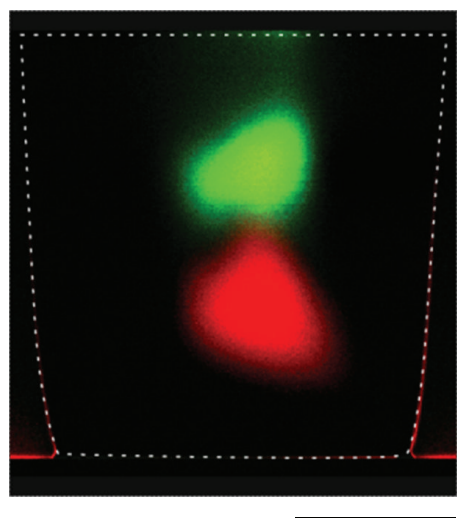

(b)

FIGURE 6: Vertically arrayed sheath flow. (a) Vertical connection of the flow shift areas. (b) Vertically arrayed 2-sample sheath flow. FITC is $1 \mu \mathrm{L} / \mathrm{min}$. Rhodamine B is $1 \mu \mathrm{L} / \mathrm{min}$. Scale bar is $50 \mu \mathrm{m}$.

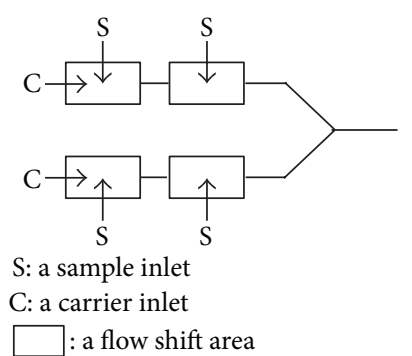

(a)

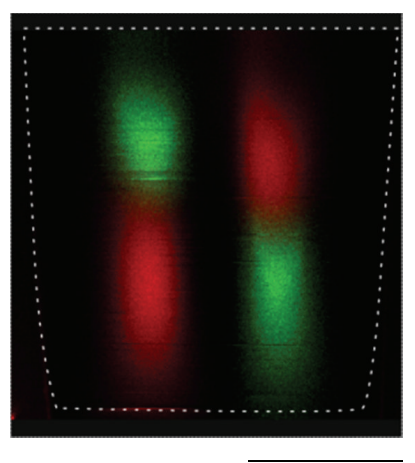

(b)

FIgURE 7: Multiple core-sheath flow. (a) Two branches of the cascade connection of the flow shift areas are connected in parallel. (b) Matrix arranged 4-sample sheath flow. FITC is $1 \mu \mathrm{L} / \mathrm{min}$. Rhodamine $\mathrm{B}$ is $1 \mu \mathrm{L} / \mathrm{min}$. Scale bar is $50 \mu \mathrm{m}$. should be designed to be two times wider than that of the branch channels.

\section{Conclusions}

Three-dimensional sheath flows were arranged in a matrix configuration. The vertical and horizontal arrays of the sample flows are key components to achieve matrix arrangement. The vertical array sheath flow was obtained by cascade connection of the flow shift areas, and the horizontal array sheath flow was obtained by parallel connection. Unlike conventional devices, the presented sheath flow microfluidic device is planar and easily fabricated without complicated alignment and bonding of several substrates containing microchannel, inlets, and outlets. Changing flow rate of sample streams, the diameter of the sample in microchannel cross section could be controlled. The matrix arrangement of the multiple sample three-dimensional sheath flow device could be potentially applicable to achieve multicomponent nanofiber by combining electrospinning. We have already developed microfluidic devices for electrospinning and are trying to find conditions to achieve multicomponent nanofibers.

\section{Acknowledgment}

This work was partly supported by Research Fellowships of the Japan Society for the Promotion of Science for Young Scientists.

\section{References}

[1] A. David, "A fine set of threads," Nature, vol. 411, p. 236, 2001.

[2] A. V. Bazilevsky, A. L. Yarin, and C. M. Megaridis, "Coelectrospinning of core-shell fibers using a single-nozzle technique," Langmuir, vol. 23, no. 5, pp. 2311-2314, 2007.

[3] A. L. Thangawng, P. B. Howell Jr., J. J. Richards, J. S. Erickson, and F. S. Ligler, "A simple sheath-flow microfluidic device for micro/nanomanufacturing: fabrication of hydrodynamically shaped polymer fibers," Lab on a Chip, vol. 9, no. 21, pp. 31263130, 2009.

[4] C. M. Hwang, A. Khademhosseini, Y. Park, K. Sun, and S. Lee, "Microfluidic chip-based fabrication of PLGA microfiber scaffolds for tissue engineering," Langmuir, vol. 24, no. 13, pp. 6845-6851, 2008.

[5] Y. Srivastava, M. Marquez, and T. Thorsen, "Microfluidic electrospinning of biphasic nanofibers with Janus morphology," Biomicrofluidics, vol. 3, no. 1, Article ID 012801, 2009.

[6] T. Lin, H. Wang, and X. Wang, "Self-crimping bicomponent nanofibers electrospun from polyacrylonitrile and elastomeric polyurethane," Advanced Materials, vol. 17, no. 22, pp. 26992703, 2005.

[7] C.-C. Chang, Z.-X. Huang, and R.-J. Yang, "Three-dimensional hydrodynamic focusing in two-layer polydimethylsiloxane (PDMS) microchannels," Journal of Micromechanics and Microengineering, vol. 17, no. 8, article 009, pp. 1479-1486, 2007.

[8] X. Mao, J. R. Waldeisen, and T. J. Huang, "Microfluidic drifting'-Implementing three-dimensional hydrodynamic focusing with a single-layer planar microfluidic device," Lab on a Chip, vol. 7, no. 10, pp. 1260-1262, 2007. 
[9] G. Hairer and M. J. Vellekoop, "An integrated flow-cell for full sample stream control," Microfluidics and Nanofluidics, vol. 7, no. 5, pp. 647-658, 2009.

[10] D. S. Kim, D. S. Kim, K. Han, and W. Yang, "An efficient 3-dimensional hydrodynamic focusing microfluidic device by means of locally increased aspect ratio," Microelectronic Engineering, vol. 86, no. 4-6, pp. 1343-1346, 2009.

[11] P. B. Howell Jr., J. P. Golden, L. R. Hilliard, J. S. Erickson, D. R. Mott, and F. S. Ligler, "Two simple and rugged designs for creating microfluidic sheath flow," Lab on a Chip, vol. 8, no. 7, pp. 1097-1103, 2008.

[12] H. Sato, Y. Sasamoto, D. Yagyu, T. Sekiguchi, and S. Shoji, "3D sheath flow using hydrodynamic position control of the sample flow," Journal of Micromechanics and Microengineering, vol. 17, no. 11, pp. 2211-2216, 2007. 

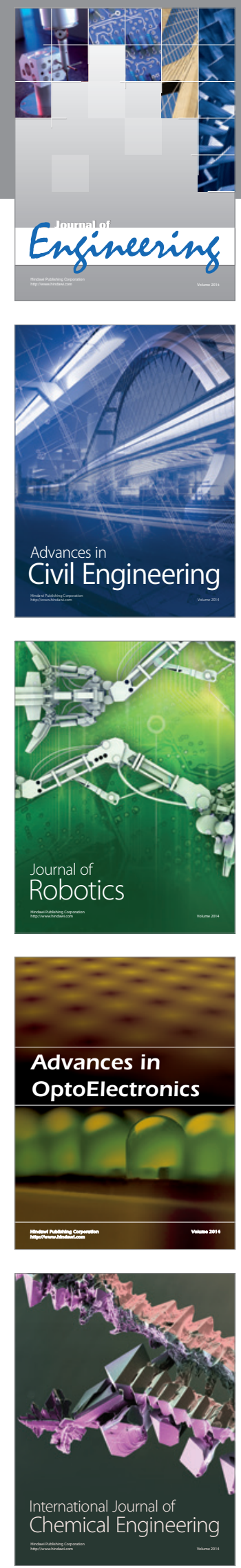

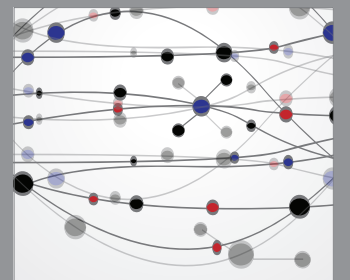

The Scientific World Journal
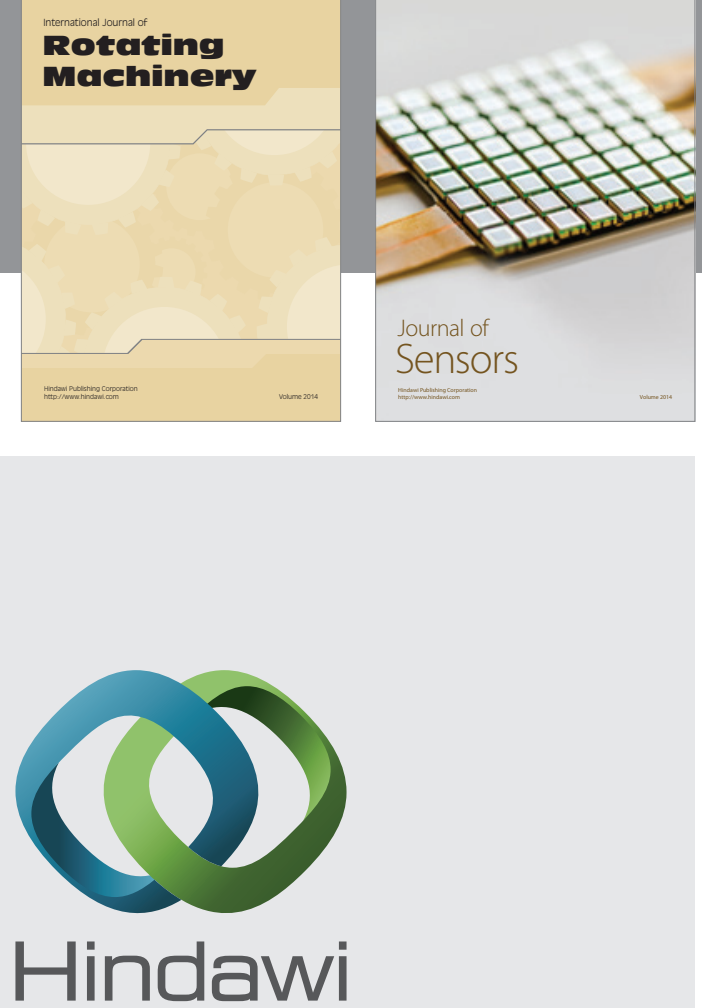

Submit your manuscripts at http://www.hindawi.com
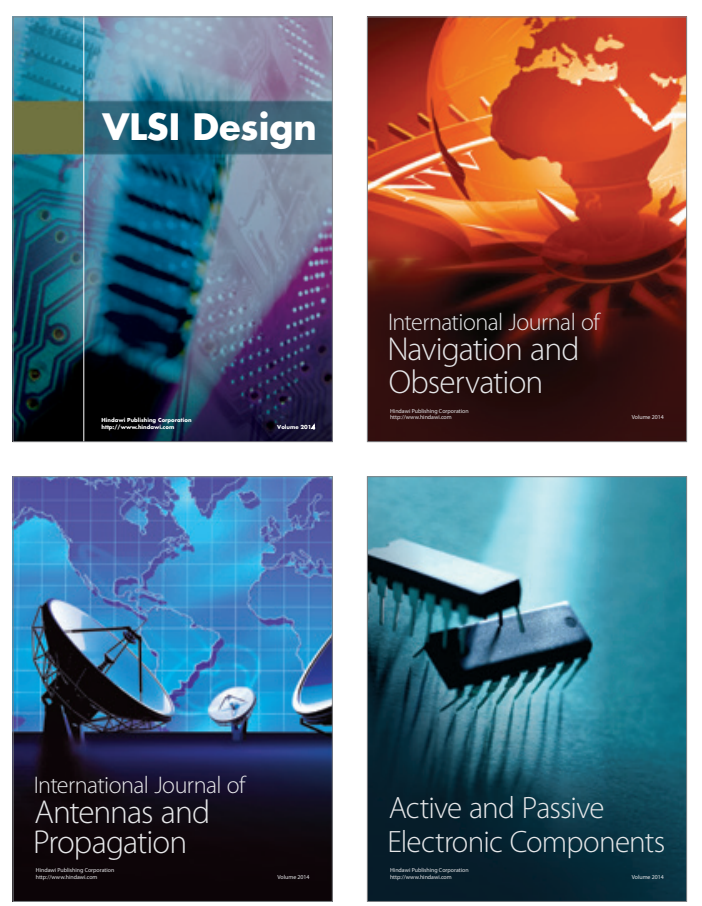
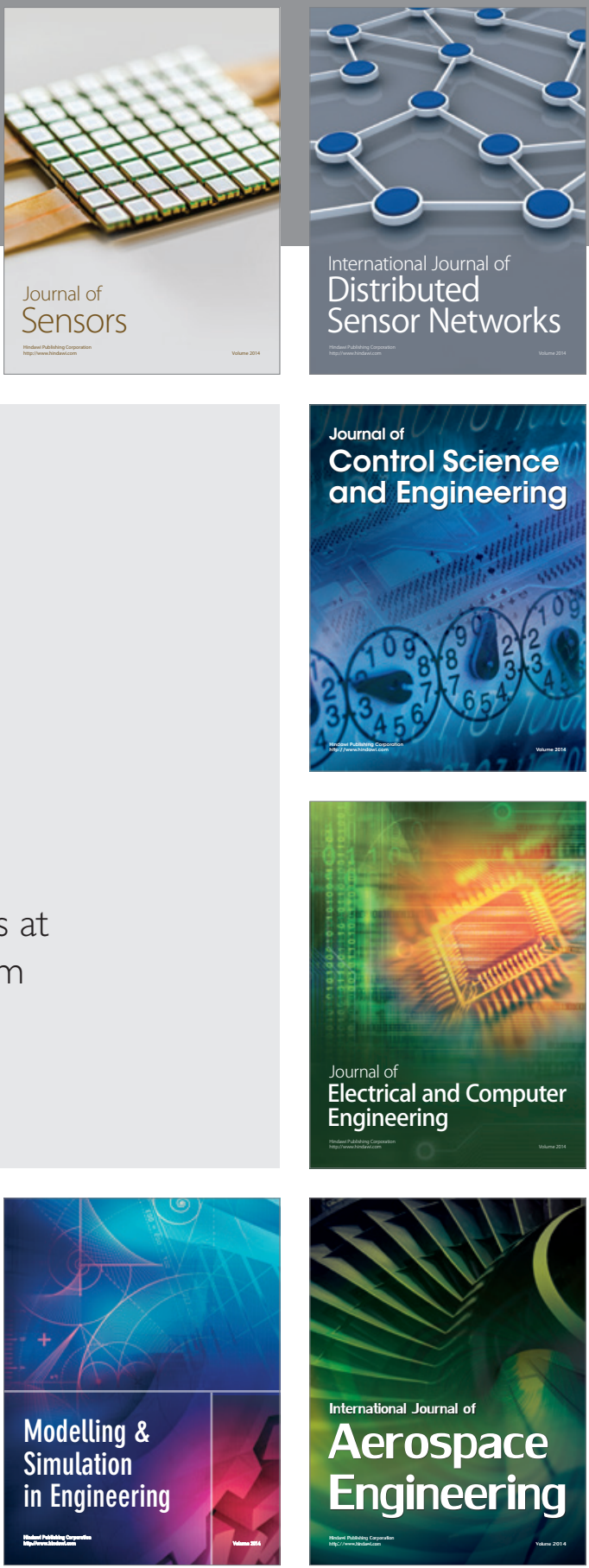

Journal of

Control Science

and Engineering
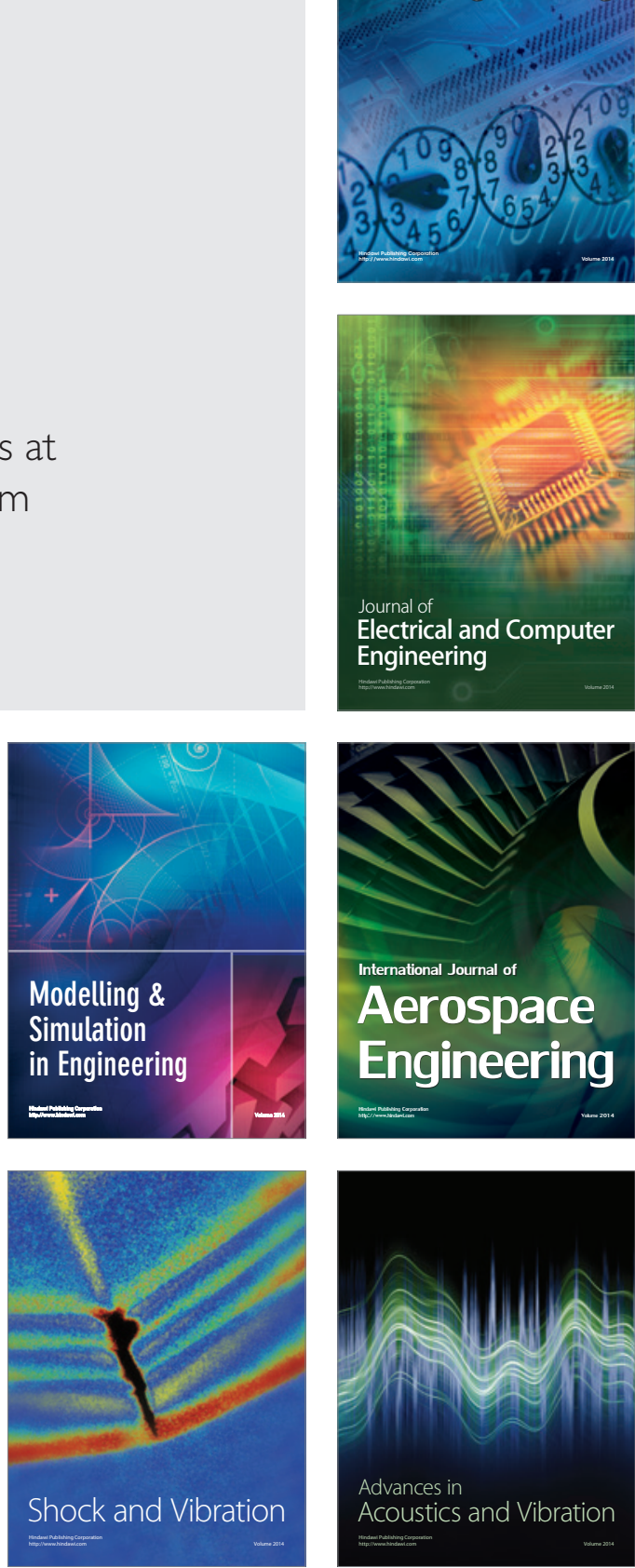